\title{
Prospective Comparative Analysis of the Incidence of Vasovagal Reaction and the Effect of Rectal Submucosal Lidocaine Injection in Stapled Hemorrhoidopexy: A Randomized Controlled Trial
}

\author{
Kyung Jin Cho ${ }^{1}$, Do Yeon Hwang ${ }^{1}$, Hyun Joo Lee ${ }^{2}$, Ki Hoon Hyun ${ }^{1}$, Tae Jung Kim ${ }^{3}$ Duk Hoon Park ${ }^{1}$ \\ Departments of ${ }^{1}$ Surgery and ${ }^{2}$ Anesthesiology, Seoul Song Do Hospital, Seoul; ${ }^{3}$ Department of Surgery, Gangseo Song Do Hospital, Seoul, Korea
}

Purpose: This study was performed to evaluate the incidence of vasovagal reactions (VVRs) and the efficacy of lidocaine injection for prevention.

Methods: One hundred seventeen patients diagnosed with hemorrhoids and scheduled to undergo a stapled hemorrhoidopexy (SH) were randomly divided according to submucosal injection to the rectum: lidocaine group ( $\mathrm{n}=53$, lidocaine injected just before full closure of the stapler) and control group $(n=58)$. Outcomes included baseline patient characteristics (American Society of Anesthesiologists physical status classification, body mass index, diabetes mellitus, hypertension, and previous VVR history), vital signs during the operation, incidence of VVRs (hypotension, bradycardia, dizziness, diaphoresis, and nausea/vomiting), and postoperative complications (pain, bleeding, and urinary retention).

Results: Baseline characteristics were similar between groups. The number of patients with lower abdominal pain after firing the stapler and incidence of dizziness were lower for the lidocaine group than for the control group (9.4\% vs. $25.9 \%$, $\mathrm{P}=0.017 ; 0 \%$ vs. $8.6 \%, \mathrm{P}=0.035$, respectively). However, there were no significant between-group differences in incidence of nausea and diaphoresis $(0 \%$ vs. $3.4 \%, \mathrm{P}=0.172)$ and syncope $(1.9 \%$ vs. $3.4 \%, \mathrm{P}=0.612)$. Fewer patients in the lidocaine group complained of postoperative pain $(41.5 \%$ vs. $58.6 \%, \mathrm{P}=0.072)$, and these patients used analgesics less frequently than those in the control group $(28.3 \%$ vs. $36.2 \%, \mathrm{P}=0.374)$.

Conclusion: Patients who received a submucosal lidocaine injection prior to $\mathrm{SH}$ experienced less lower abdominal pain and dizziness compared with those who received standard treatment. A larger, more detailed prospective study is needed for further analysis.

Keywords: Vasovagal syncope; Stapled hemorrhoidopexy; Local anesthetics; Randomized controlled trial

\section{INTRODUCTION}

Stapled hemorrhoidopexy (SH) was introduced by Longo in 1998

Received: Dec 24, 2019 - Revised: Feb 11, 2020 - Accepted: Feb 12, 2020

Correspondence to: Duk Hoon Park, M.D.

Department of Surgery, Seoul Song Do Hospital, 78 Dasan-ro, Jung-gu, Seoul 04597, Korea

Tel: +82-2-2250-7368, Fax: +82-2-2233-8528

E-mail: parkx0642@naver.com

ORCID: https://orcid.org/0000-0003-2531-2871

(C) 2020 The Korean Society of Coloproctology

This is an open-access article distributed under the terms of the Creative Commons Attribution NonCommercial License (https://creativecommons.org/licenses/by-nc/4.0) which permits unrestricted noncommercial use, distribution, and reproduction in any medium, provided the original work is properly cited. as a minimally invasive new technique for treating hemorrhoidal disease [1]. Instead of submucosal excision of the prolapsed hemorrhoidal pedicles, a stapling device is used to reposition the prolapsed hemorrhoids, which involves simultaneous circumferential excision and anastomosis [2, 3]. Compared with conventional hemorrhoidectomy, this approach is advantageous because of less postoperative pain, shorter hospital stay, and shorter duration of convalescence; these improvements in patient outcomes have resulted in widespread use of SH by coloproctologists [4-6]. Although the procedure is generally well-tolerated, some patients experience pain, and there is risk of a vasovagal reaction (VVR).

VVR is also referred to as neurocardiogenic or neuromediated syndrome because the usual cause stems from the nerves of the 
heart, are relatively frequent during medical interventions, and pose a challenge to physicians, with an annual incidence of 1.3 to 2.7 events per 1,000 individuals [7]. The incidence of VVRs in interventional cardiology procedures is between $3.4 \%$ and $13.9 \%$ [8], and in colonoscopy, this rate varies between $0.2 \%$ and $0.5 \%$ with a mortality rate of $0.03 \%$ to $0.05 \%$ [9]. VVR occurred in $16 \%$ of patients with conventional hemorrhoidectomy, one of the most common procedures performed by coloproctologists, and $0.6 \%$ in rubber band ligation $[10,11]$. The presence of pain, tissue injury, and strong emotional reactions may contribute to development of VVR $[12,13]$. In patients with a mild pathology, the symptoms are limited to presyncope or aura (dizziness, mental confusion, weakness, and diaphoresis) without loss of consciousness. Most patients do not experience transient loss of consciousness $[14,15]$. Though it is frequently benign, a malignant form of this disorder with episodes of prolonged cardioinhibition has been reported; these can often be caused by surgery. These episodes can culminate in asystole and, if not interrupted, may simulate a sudden cardiac deathlike episode [16, 17].

It has been suggested that use of local anesthesia may reduce pain or prevent VVR. Several reports have been published on the effect of local anesthetic injection on the rectal mucosa during open hemorrhoidectomy and rubber band ligation $[10,11]$. However, no published clinical studies have discussed the incidence or prevention of VVR during SH. To address this, we conducted a prospective randomized controlled trial (RCT) aimed to analyze the incidence of VVR and the effect of rectal submucosal lidocaine injection in $\mathrm{SH}$.

\section{METHODS}

The trial was approved by the Institutional Review Board of Seoul Song Do Hospital (No. 2018-005), and all patients provided written informed consent to participate in the study. Between May and October 2018, the study enrolled 117 patients diagnosed with hemorrhoids and scheduled to undergo SH. Patients with a his-

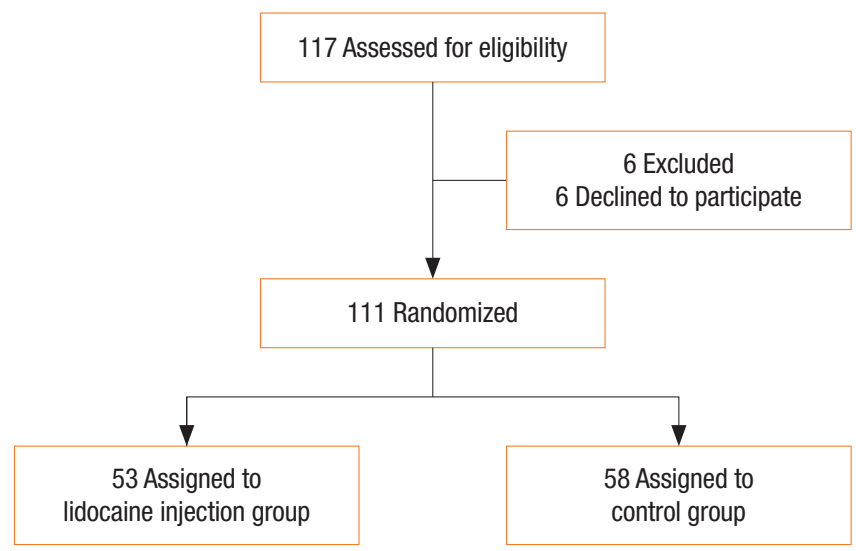

Fig. 1. Flow diagram showing the study design. tory of inflammatory bowel disease (e.g., Crohn disease or ulcerative colitis), tuberculosis, or coexisting anorectal diseases such as anal fistula and anal fissure and those who requested a sedative agent during the procedure were also excluded. Six patients were excluded as they declined to participate in the study (Fig. 1).

Patients were randomly assigned by a computer-generated list to either the lidocaine group, which received a local anesthetic injection during the procedure, or the control group with no injection. Allocations were sealed in opaque, numbered envelopes. All patients received spinal anesthesia administered by a single anesthesiologist $(0.5 \%$ heavy bupivacaine $0.05 \mathrm{mg} / \mathrm{cm}$ injected by a 25 gage spinal needle on the L2-3 interspace). The procedures were performed by 2 colorectal surgeons using 2 stapler devices, $\mathrm{PPH}$ 33 (DAVID, Ningbo, China) and TST33-S180 (Touchstone, Suzhou, China). At completion of purse-string suture and before firing of the device, the patients in the lidocaine group received 5\% lidocaine in the rectal submucosal area to be stapled (Fig. 2).

During circular SH, $1 \mathrm{~mL}$ of lidocaine was injected around the anus at each of the 12, 3, 6, and 9 oclock positions. During partial $\mathrm{SH}, 1 \mathrm{~mL}$ of lidocaine was injected onto each of the 3 rectal mucosae protruding through the circular anal dilator window. At each step of the procedure (before the operation, after the closure by the stapler, after firing, and after completion of the procedure), vital signs (systolic pressure, diastolic pressure, and pulse rate) were measured and recorded by the circulating nurse. During the procedure, the surgeons and anesthesiologist monitored the patients for complications such as lower abdominal pain, dizziness, nausea, diaphoresis, and syncope.

After the surgery, patients were transferred to the general ward, and pain was recorded at 1 hour, 6 hours, and 24 hours postoperatively using a visual analogue scale (VAS; 1 to 10). Patients were reviewed for complications including bleeding and urinary retention. Patients with pain and those with a VAS score greater than 4 were given injections of 10 to $30 \mathrm{mg}$ of ketorolac.

Statistical analysis was completed using IBM SPSS Statistics ver. 23.0 (IBM Corp., Armonk, NY, USA). Categorical data were ana-

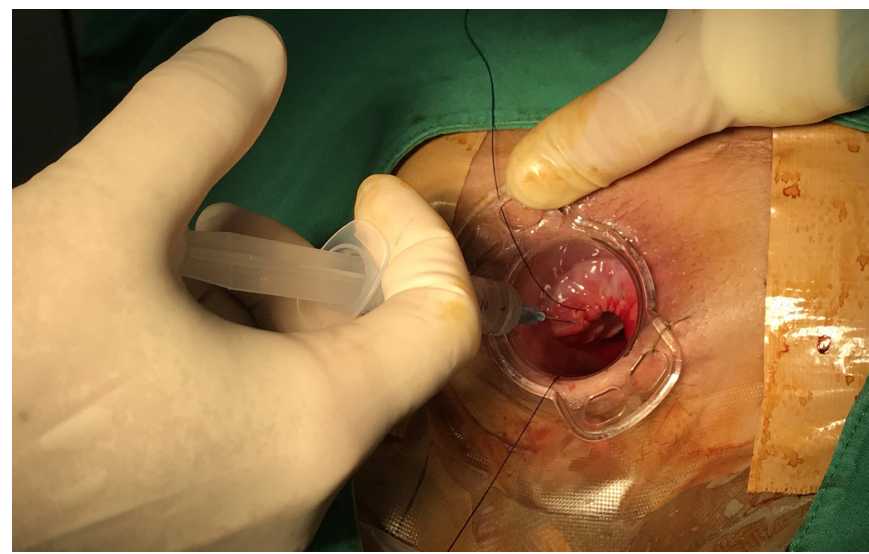

Fig. 2. Submucosal lidocaine injection. 


\section{Coloproctology}

lyzed using the chi-square test, and continuous data were analyzed using Student t-test. Data were expressed as mean \pm standard deviation or number (percentage). $\mathrm{P}<0.05$ was considered statistically significant.

\section{RESULTS}

There were no significant between-group differences in baseline characteristics (Table 1). Comparison of the monitored vital signs (systolic pressure, diastolic pressure, and pulse rate) revealed no significant difference between the 2 groups (Table 2). The rate of intraoperative complications, such as lower abdominal pain $(9.4 \%$ [ 5 of 53 ] vs. $25.9 \%$ [ 15 of 58 ], $\mathrm{P}=0.017$ ) and dizziness ( $0 \%$ [ 0 of

Table 1. Baseline characteristics of the patients

\begin{tabular}{|c|c|c|c|}
\hline Characteristic & $\begin{array}{l}\text { Lidocaine group } \\
\qquad(\mathrm{n}=53)\end{array}$ & $\begin{array}{c}\text { Control group } \\
(n=58)\end{array}$ & P-value \\
\hline Age (yr) & $50.8 \pm 20.1$ & $50.2 \pm 19.5$ & 0.639 \\
\hline \multicolumn{4}{|l|}{ Sex } \\
\hline Male & $37(69.8)$ & 44 (75.9) & 0.374 \\
\hline Female & $16(30.2)$ & $14(24.1)$ & 0.508 \\
\hline Body mass index $\left(\mathrm{kg} / \mathrm{m}^{2}\right)$ & $23.7 \pm 4.2$ & $24.7 \pm 3.8$ & 0.412 \\
\hline ASA PS classification & & & 0.385 \\
\hline I & $30(56.6)$ & $34(58.6)$ & \\
\hline$\|$ & $22(41.5)$ & 21 (36.2) & \\
\hline III & $1(1.9)$ & $3(5.2)$ & \\
\hline Alcohol (g/day) & $6.0 \pm 11.9$ & $4.5 \pm 10.9$ & 0.516 \\
\hline Smoking (pack-yr) & $1.1 \pm 6.0$ & $2.0 \pm 5.9$ & 0.206 \\
\hline \multicolumn{4}{|l|}{ Preexisting disease } \\
\hline Hypertension & 17 (32.1) & $14(24.1)$ & 0.352 \\
\hline Diabetes & $4(7.5)$ & $5(8.6)$ & 0.836 \\
\hline $\mathrm{BPH}$ & $5(9.4)$ & $4(6.9)$ & 0.625 \\
\hline Hyperlipidemia & $6(11.3)$ & $11(19.0)$ & 0.264 \\
\hline $\begin{array}{l}\text { Cardiovascular } \\
\text { (angina, stroke, MI) }\end{array}$ & $1(1.9)$ & $4(6.9)$ & 0.204 \\
\hline Syncope & $1(1.9)$ & 0 & 0.293 \\
\hline Hemorrhoid grade & & & 0.099 \\
\hline$\|$ & $1(1.9)$ & $7(12.1)$ & \\
\hline III & $35(66.0)$ & $37(63.8)$ & \\
\hline IV & $17(32.1)$ & $14(24.1)$ & \\
\hline \multicolumn{4}{|c|}{ Preoperative manometry (maximal, mmHg) } \\
\hline Resting & $74.7 \pm 18.4$ & $75.0 \pm 19.8$ & 0.983 \\
\hline Voluntary contraction & $227.6 \pm 49.3$ & $230.0 \pm 27.5$ & 0.749 \\
\hline Squeezing & $152.2 \pm 50.1$ & $153.8 \pm 29.3$ & 0.841 \\
\hline
\end{tabular}

Values are presented as mean \pm standard deviation or number (\%).

ASA, American Society of Anesthesiologists; PS, physical status; BPH, benign prostate hypertrophy; Ml, myocardial infarction.
53 ] vs. $8.6 \%$ [ 5 of $58 \%$ ], $\mathrm{P}=0.035$ ) was significantly lower in the lidocaine group than in the control group (Table 3). Additional

Table 2. Patient vital signs during procedures

\begin{tabular}{lccc}
\hline Variable & $\begin{array}{c}\text { Lidocaine group } \\
(\mathrm{n}=53)\end{array}$ & $\begin{array}{c}\text { Control group } \\
(\mathrm{n}=58)\end{array}$ & P-value \\
\hline Baseline & & & \\
SP (mmHg) & $127.93 \pm 15.80$ & $129.94 \pm 14.14$ & 0.375 \\
DP (mmHg) & $76.03 \pm 12.85$ & $78.89 \pm 12.32$ & 0.751 \\
PR (beats/min) & $75.58 \pm 12.37$ & $78.08 \pm 11.68$ & 0.638 \\
Closure & & & \\
SP (mmHg) & $128.00 \pm 13.45$ & $127.23 \pm 12.81$ & 0.596 \\
DP (mmHg) & $84.88 \pm 11.22$ & $81.96 \pm 11.14$ & 0.841 \\
PR (beats/min) & $75.45 \pm 13.42$ & $76.39 \pm 12.23$ & 0.566 \\
Firing & & & \\
SP (mmHg) & $130.14 \pm 14.79$ & $128.34 \pm 16.06$ & 0.458 \\
DP (mmHg) & $82.07 \pm 10.95$ & $80.96 \pm 9.83$ & 0.433 \\
PR (beats/min) & $75.22 \pm 12.77$ & $76.66 \pm 11.71$ & 0.530 \\
Postoperative & & & \\
SP (mmHg) & $128.81 \pm 14.20$ & $127.40 \pm 13.70$ & 0.673 \\
DP (mmHg) & $79.72 \pm 11.26$ & $79.51 \pm 10.18$ & 0.739 \\
PR (beats/min) & $77.00 \pm 11.04$ & $76.72 \pm 11.53$ & 0.523 \\
\hline
\end{tabular}

Values are presented as mean \pm standard deviation.

$\mathrm{SP}$, systolic pressure; DP, diastolic pressure; PR, pulse rate.

Table 3. Complications and pain scores in the 2 groups

\begin{tabular}{lccc}
\hline Variable & $\begin{array}{c}\text { Lidocaine group } \\
(\mathrm{n}=53)\end{array}$ & $\begin{array}{c}\text { Control group } \\
(\mathrm{n}=58)\end{array}$ & P-value \\
\hline Intraoperative & $5(9.4)$ & $15(25.9)$ & 0.017 \\
\hline Lower abdominal pain & $0(0)$ & $5(8.6)$ & 0.035 \\
Dizziness & $0(0)$ & $2(3.4)$ & 0.172 \\
Nausea & $0(0)$ & $2(3.4)$ & 0.172 \\
Diaphoresis & $1(1.9)$ & $2(3.4)$ & 0.612 \\
Syncope & & & \\
Postoperative & $22(41.5)$ & $34(58.6)$ & 0.072 \\
\hline Operation site pain & $3(5.7)$ & $6(10.3)$ & 0.366 \\
Urinary retention & $1(1.9)$ & $2(3.4)$ & 0.612 \\
Bleeding & & & \\
Postoperative VAS & $0.40 \pm 0.66$ & $0.53 \pm 0.77$ & 0.220 \\
\hline hr & $3.00 \pm 1.68$ & $3.31 \pm 1.83$ & 0.237 \\
6 hr & $2.29 \pm 0.97$ & $2.14 \pm 1.17$ & 0.448 \\
\hline 24 hr & $15(28.3)$ & $21(36.2)$ & 0.374 \\
\hline Analgesia use & & & \\
\hline
\end{tabular}

Values are presented as number (\%) or mean \pm standard deviation. VAS, visual analogue scale. 
nonsignificant differences in rates of intraoperative and postoperative complications were smaller in the lidocaine group. Postoperative VAS scores were nonsignificantly different between the 2 groups.

\section{DISCUSSION}

VVRs during surgery are relatively frequent, cause discomfort to patients, and can pose a clinical challenge for physicians. Several methods have been proposed to solve this problem, most of which depend on the experience of physicians. There is little-tono published research or data available regarding VVR in SH. This RCT evaluated the incidence of VVR during SH for the first time and analyzed the effect of rectal submucosal lidocaine injection on this incidence rate.

In general, VVR has 3 subsequent symptomatologic phases. The first phase is presyncope, or aura, with premonitory symptoms (dizziness, mental confusion, weakness, sweating). Second is loss of consciousness, with possible convulsions caused by anoxia (oxygen deprivation), which is generally referred to as syncope. Third, the postsyncopal period is usually characterized by recovery of consciousness and orientation [18].

Stimulation of afferent vagal nerves can occur from either the heart or from other anatomical structures, including the great vessels (carotid bulb), eyes (oculocardiac reflex), and viscera such as gastrointestinal tract and urinary bladder [19]. Pain due to mesenteric stretching and colonic distension can trigger increases in vagal tone [20]. In an RCT, Kim et al. [10] reported that submucosal lidocaine injection significantly reduced VVRs such as nausea, vomiting, sweating, and dizziness following lower abdominal pain at open hemorrhoidectomy. In another randomized trial, Kwok et al. [11] concluded that injection of submucosal bupivacaine reduced pain during and after rubber band ligation hemorrhoidectomy.

Therefore, we hypothesized that pain is likely to occur during the surgery, especially when the stapler is closed fully; pain during stapler firing may trigger VVR. This study found that, while the incidence of syncope was not significantly different between the 2 groups, the incidence of dizziness corresponding to presyncope was significantly different, and this corresponded with a significant between-group difference in pain.

There were some limitations to this study, including the small sample size and the difficultly in accurately determining whether pain reduction during surgery was due to the anesthetic effect of lidocaine or bulging of the rectal mucosa. Further study is needed to determine the effects of injecting a substance other than lidocaine. Reclassifying the 2 groups according to presence of pain can provide a more accurate understanding of the cause and prevention of pain and in identifying the most common and important cause of VVR. The randomization protocol did not consider the type of SH (circular or partial) or the grade of hemorrhoids. Given that partial SH preserves more normal mucosa than circu- lar SH and that higher grade of hemorrhoid was associated with larger amount of tissue resection during $\mathrm{SH}$, future studies on the amount of rectal mucosa should consider specific operation type and hemorrhoid grade.

To the best of our knowledge, this study is the first to prospectively analyze the incidence of VVR during $\mathrm{SH}$. Our results show that injecting lidocaine into the rectal submucosa may have positive effects on prevention of VVRs.

\section{CONFLICT OF INTEREST}

No potential conflict of interest relevant to this article was reported.

\section{REFERENCES}

1. Ommer A, Hinrichs J, Mollenberg H, Marla B, Walz MK. Longterm results after stapled hemorrhoidopexy: a prospective study with a 6-year follow-up. Dis Colon Rectum 2011;54:601-8.

2. Longo A. Treatment of hemorrhoids disease by reduction of mucosa and hemorrhoidal prolapse with a circular stapler suturing device: a new procedure. In: European Association for Endoscopic Surgery. Proceedings of the 6th World Congress of Endoscopic Surgery; 1998 Jun 3-6; Rome, Italy. Bologna: Monduzzi Editore; 1998. p. 777-84.

3. Lohsiriwat V. Treatment of hemorrhoids: a coloproctologist's view. World J Gastroenterol 2015;21:9245-52.

4. Gabrielli F, Chiarelli M, Cioffi U, Guttadauro A, De Simone M, Di Mauro P, et al. Day surgery for mucosal-hemorrhoidal prolapse using a circular stapler and modified regional anesthesia. Dis Colon Rectum 2001;44:842-4.

5. Michalik M, Pawlak M, Bobowicz M, Witzling M. Long-term outcomes of stapled hemorrhoidopexy. Wideochir Inne Tech Maloinwazyjne 2014;9:18-23.

6. Bellio G, Pasquali A, Schiano di Visconte M. Stapled hemorrhoidopexy: results at 10-year follow-up. Dis Colon Rectum 2018; 61:491-8.

7. Savage DD, Corwin L, McGee DL, Kannel WB, Wolf PA. Epidemiologic features of isolated syncope: the Framingham Study. Stroke 1985;16:626-9.

8. Juergens CP, Lo S, French JK, Leung DY. Vaso-vagal reactions during femoral arterial sheath removal after percutaneous coronary intervention and impact on cardiac events. Int J Cardiol 2008;127:252-4.

9. Manta R, Tremolaterra F, Arezzo A, Verra M, Galloro G, Dioscoridi L, et al. Complications during colonoscopy: prevention, diagnosis, and management. Tech Coloproctol 2015;19:505-13.

10. Kim TS, Kim DS, Kang YS, Jung SY, Cho HJ, Lee DH. Effect of lidocaine injected on lower rectal submucosa during hemorrhoidectomy under caudal anesthesia. J Korean Soc Coloproctol 1998; 14:85-9.

11. Kwok HC, Noblett SE, Murray NE, Merrie AE, Hayes JL, Bissett 


\section{Coloproctology kyung Jin Cho, et al.}

IP. The use of local anaesthesia in haemorrhoidal banding: a randomized controlled trial. Colorectal Dis 2013;15:487-91.

12. Adlakha H, Gupta R, Hassan R, Kern JH. Association between baseline blood pressures, heart rates, and vasovagal syncope in children and adolescents. Cureus 2018;10:e2119.

13. Boss BJ, Brewer L. Syncope: neuroscience nursing assessment based on an understanding of underlying pathophysiological mechanisms. J Neurosci Nurs 1988;20:245-52.

14. Aydin MA, Salukhe TV, Wilke I, Willems S. Management and therapy of vasovagal syncope: a review. World J Cardiol 2010;2: 308-15.

15. Jardine DL, Wieling W, Brignole M, Lenders JWM, Sutton R, Stewart J. The pathophysiology of the vasovagal response. Heart Rhythm 2018;15:921-9.

16. Brignole M, Moya A, de Lange FJ, Deharo JC, Elliott PM, Fanci- ulli A, et al. 2018 ESC Guidelines for the diagnosis and management of syncope. Kardiol Pol 2018;76:1119-98.

17. Khera S, Palaniswamy C, Aronow WS, Sule S, Doshi JV, Adapa S, et al. Predictors of mortality, rehospitalization for syncope, and cardiac syncope in 352 consecutive elderly patients with syncope. J Am Med Dir Assoc 2013;14:326-30.

18. Occhetta E, Audoglio R. Malignant vasovagal syncope: physiopathology, diagnosis, epidemiology, and medical treatment. Prog Biomed Res 2003;8:174-84.

19. Sharma G, Boopathy Senguttuvan N, Juneja R, Kumar Bahl V. Neurocardiogenic syncope during a routine colonoscopy: an uncommon malignant presentation. Intern Med 2012;51:891-3.

20. Chen-Scarabelli C, Scarabelli TM. Neurocardiogenic syncope. BMJ 2004;329:336-41. 